\title{
Multigrid meets neural nets
}

\author{
M. Bäker, G. Mack and M. Speh \\ II. Institut für Theoretische Physik, Universität Hamburg, Luruper Chaussee 149, 2000 Hamburg 50
}

We present evidence that multigrid (MG) works for wave equations in disordered systems, e.g. in the presence of gauge fields, no matter how strong the disorder. We introduce a "neural computations" point of view into large scale simulations: First, the system must learn how to do the simulations efficiently, then do the simulation (fast). The method can also be used to provide smooth interpolation kernels which are needed in multigrid Monte Carlo updates.

\section{INTRODUCTION}

There is a stochastic multigrid method and a deterministic one. The stochastic version is used to compute high dimensional integrals in Euclidean quantum field theory or statistical mechanics by a Monte Carlo method which uses updates at different length scales [1,2]. The deterministic version [3] solves discretized partial differential equations. One hopes to use both of them in simulations of lattice QCD, for updating the gauge fields and for computing fermion propagators in given gauge fields. In either case the aim is to beat critical slowing down (CSD) in nearly critical systems.

Our notation is as follows: $\Lambda^{0}$ denotes a given "fundamental" lattice $\Lambda^{0}$ of spacing $a_{0}$. Coarser (block) lattices of increasing spacings $a_{j}=L_{b}^{j} a_{0}$ are denoted $\Lambda^{1}, \Lambda^{2}, \ldots, \Lambda^{N}$. Typically, we chose $L_{b}=2$, and a single point as the last layer $\Lambda^{N}$. Interpolation operators $\mathcal{A}^{j}$ are introduced to transfer functions on coarser lattices into functions on finer lattices, while restriction operators $C^{j} \equiv \mathcal{A}^{j *}$ transfer functions from finer to coarser lattices ("variational coarsening").

\section{IMPORTANCE OF SMOOTHNESS}

A crucial problem is how to define and exhibit smooth functions in the disordered context, i.e. when translation symmetry is strongly violated. Other possible applications besides gauge theories are low lying states of spin glasses, the shape of a lightning, waves on fractal lattices (with bond percolation), or the localization of low lying electronic states in amorphous materials.

In the case of deterministic MG, one wants to solve a discretized elliptic differential equation on $\Lambda^{0}$ :

$D_{0} \xi^{0}=f^{0}$.

It might have arisen from an eigenvalue equation $D_{0} \xi^{0}=\varepsilon \xi^{0}$ by inverse iteration. If $D_{0}$ has a small eigenvalue, then local relaxation algorithms suffer from CSD. After some relaxation sweeps on $\Lambda^{0}$ one gets an approximate solution $\widetilde{\xi}^{0}$ whose error $e^{0}=\xi^{0}-\widetilde{\xi}^{0}$ is not necessarily small but is smooth (on length scale $a_{0}$ ). The unknown error $e^{0}$ satisfies the equation

$D_{0} e^{0}=r^{0}$.

with the residual $r^{0}=f^{0}-D_{0} \widetilde{\xi}^{0}$. Given that $e^{0}$ is smooth, it can be obtained by smooth interpolation of a suitable function $e^{1}$ on $\Lambda^{1}$,

$e^{0}=\mathcal{A}^{1} e^{1}$.

That is, $e_{z}^{0}=\sum_{x \in \Lambda^{1}} \mathcal{A}_{z x}^{1} e_{x}^{1}$ with $\mathcal{A}_{z x}^{1}$ which depends smoothly on $z$. Now define a restriction operator $C^{1}$ such that $C^{1} \mathcal{A}^{1}=1$. Then (3) can be inverted, $e^{1}=C^{1} e^{0}$. Applying $C^{1}$ to both sides of (2) yields an equation for $e^{1}$,

$D_{1} e^{1}=r^{1}$,

with $r^{1}=C^{1} r^{0}$ and the effective operator $D_{1}=$ $C^{1} D_{0} \mathcal{A}^{1}$. Given $e^{1}$, one obtains $e^{0}$ from (3), and $\widetilde{\xi}^{0}+e^{0}$ is an improved solution of (11). Thus, the problem has been reduced to an equation on the 
lattice $\Lambda^{1}$ which has fewer points. If necessary, one repeats the procedure, moving to $\Lambda^{2}$ etc. The procedure stops, because an equation on a "lattice" $\Lambda^{N}$ with only a single point is easy to solve.

The iterated interpolation $\mathcal{A}^{[0 j]} \equiv \mathcal{A}^{1} \mathcal{A}^{2} \ldots \mathcal{A}^{j}$ from $\Lambda^{j}$ to $\Lambda^{0}$ should yield functions on $\Lambda^{0}$ which are smooth on length scale $a_{j}$, i.e. which change little over a distance $a_{j}$ (in the ordered case). For reasons of practicality, one must require $\mathcal{A}_{z x}^{j}=0$ unless $z$ is near $x$.

\section{SMOOTHNESS AND DISORDER}

A successful MG scheme, whether deterministic or stochastic, needs smooth interpolation kernels $\mathcal{A}$. Thus we may ask: Which functions are smooth in the disordered situation, for instance in an external gauge field?

A (gauge covariant) naive answer is

$\sum_{\mu}\left(\nabla_{\mu} \xi, \nabla_{\mu} \xi\right)=(\xi,-\Delta \xi) \geq \varepsilon_{0}(\xi, \xi)$

(with discretized covariant derivatives $\nabla_{\mu}$ ). By definition, the lowest eigenvalue $\varepsilon_{0}$ of the negative covariant Laplacian $-\Delta$ is not small for disordered gauge fields. (It is positive and vanishes only for pure gauges.) Therefore there are no smooth functions in this case.

Nevertheless there is an answer to the question, assuming a fundamental differential operator $D_{0}$ is specified by the problem (in the stochastic case, the Hamiltonian often provides $\left.D_{0}\right)$ :

$A$ function $\xi$ on $\Lambda^{0}$ is smooth on length scale a when $\left\|D_{0} \xi\right\|^{2} \ll\|\xi\|^{2}$ in units $a=1$.

We found that a deterministic multigrid which employs interpolation kernels $\mathcal{A}^{[0 j]}$ from $\Lambda^{j}$ to the fundamental lattice $\Lambda^{0}$ which are smooth in this sense, works for arbitrarily disordered gauge fields. When there are no smooth functions in this sense at length scale $a_{0}$, then $D_{0}$ has no low eigenvalue, and there is no CSD and no need for MG.

The above answer appears natural, and the "projective MG" of [., is in its spirit. But to obtain kernels $\mathcal{A}_{z x}^{[0 j]}$ which are smooth on length scale $a_{j}$, one needs approximate solutions of eigenvalue equations

$D_{0} \mathcal{A}_{z x}^{[0 j]}=\varepsilon_{0}(x) \mathcal{A}_{z x}^{[0 j]}$
Since $\mathcal{A}^{[0 j]}$ is required to vanish for $z$ outside a neighbourhood of $x$, the problem involves Dirichlet boundary conditions. For large $j, \mathcal{A}^{[0 j]}$ will have a large support. If there is no degeneracy in the lowest eigenvalue, one can use inverse iteration combined with standard relaxation algorithms for the resulting inhomogeneous equation. But this and other standard methods will suffer from CSD again. Moreover, in the standard multigrid setup, one uses basic interpolation kernels $\mathcal{A}^{j}$ which interpolate from one grid $\Lambda^{j}$ to the next finer one. In this case

$\mathcal{A}^{[0 j]}=\mathcal{A}^{1} \mathcal{A}^{2} \ldots \mathcal{A}^{j}$,

and (5) becomes a very complicated set of nonlinear conditions. Possible solutions are

(i) Replace (5) by minimality of a cost functional (cp. later). Use neural algorithms to find kernels $\mathcal{A}^{j}$ which minimize it. This is still under study.

(ii) Give up factorization (6) and determine independent kernels $\mathcal{A}^{[0 j]}$ as solutions of (5) by multigrid iteration. This is done successively for $j=1,2, \ldots$ One uses already determined kernels $\mathcal{A}^{[0 k]}$ with $k<j$ for updating $\mathcal{A}^{[0 j]}$. We found that this works very well - cp. sect. 6.

Method (ii) will need of order $L^{d} \ln L$ storage space and $L^{d} \ln ^{2} L$ computational work for a $d$ dimensional system of linear extension $L$.

\section{CRITERIA FOR OPTIMALITY}

Any iteration to solve (11) amounts to updating steps of the form

$\widetilde{\xi}^{0} \mapsto \widetilde{\xi}^{0 \prime}=\varrho \widetilde{\xi}^{0}+\sigma f^{0}$.

with the iteration matrix $\varrho$ whose norm governs the convergence, and $\sigma=(1-\varrho) D_{0}^{-1}$. If $\|\varrho\|<$ 1 , the iteration converges with a relaxation time $\tau \leq-1 / \ln \|\varrho\|$. Parameters in the algorithm such as operators $\mathcal{A}_{z x}^{j}, C_{x z}^{j}$ and $D_{j}$ - are optimal if the cost functional $E=\|\varrho\|^{2}$ is at its minimum.

As an example, consider a twogrid iteration in which a standard relaxation sweep on $\Lambda^{0}$ with iteration matrix $\varrho_{0}$ is followed by exact solution 
of the coarse grid equation (伍). The second step leads to an updating with some iteration matrix $\varrho_{1}$, and $\varrho=\varrho_{1} \varrho_{0}$. Therefore one may estimate $E \leq\left\|D_{0} \varrho_{0}\right\|^{2} E_{1}$ with $E_{1}=\left\|D_{0}^{-1} \varrho_{1}\right\|^{2}$ (fine grid relaxation smoothens the error but does not converge fast - therefore $\left\|D_{0} \varrho_{0}\right\|$ is suppressed whereas $\left\|\varrho_{0}\right\|$ is not much smaller than 1 ) and try to optimize the parameters above by minimizing $E_{1}$ : Using the trace norm, $\|\varrho\|^{2}=\operatorname{tr} \varrho \varrho^{*}$, one finds

$E_{1}=$ Volume $^{-1} \sum_{z, w \in \Lambda^{0}}\left|\Gamma_{z w}\right|^{2}$

with $\Gamma=D_{0}^{-1}-\mathcal{A}^{1} D_{1}^{-1} C^{1}$. Prescribing $C^{1}$, and determining $D_{1}$ and $\mathcal{A}^{1}$ by minimizing $E_{1}$ yields what we call the "ideal interpolation kernel" $\mathcal{A}_{z x}^{1}$ for a given restriction map $C^{1}$. Since it has exponential tails instead of vanishing for $z$ outside a neighbourhood of $x$, it is impractical for production runs, though [6].

\section{NEURAL MULTIGRID (NMG)}

A feed-forward artificial neural network (ANN) [7] can perform the computations to solve (2) by MG relaxation.

The nodes ("neurons") of the NMG are identified with points of the MG as shown in Fig. 1 The resulting NMG consists of two copies of the same MG, except that the last layer $\Lambda^{N}$ is not duplicated. In the standard MG approach, the basic interpolation kernels $\mathcal{A}^{j}$ interpolate from one layer $\Lambda^{j}$ to the preceding one, $\Lambda^{j-1}$. Each node is connected to some of the nodes in the preceding layer. In the upper half, the connection strength from $x \in \Lambda^{j}$ to $z \in \Lambda^{j-1}$ is $\mathcal{A}_{z x}^{j}$. In the lower half, node $z \in \Lambda^{j-1}$ is connected to $x \in \Lambda^{j}$ with strength $R_{x z}^{j}$. In addition there is a connection of strength $\omega_{j} d_{j}^{-1}$ between the two nodes which represent the same point $z$ in $\Lambda^{j}(j<N)$.

According to Hebb's hypothesis of synaptical learning, a biological neural network learns by adjusting the strength of its synaptical connections.

The network receives as input an approximate solution $\xi$ of (2.1), from which the residual $r^{0}=$ $f^{0}-D_{0} \xi$ is then determined. It computes as output an improved solution $O=\xi+\delta \xi$. The desired output ("target") is $\zeta=D_{0}^{-1} f^{0} . \delta \xi$ is a linear

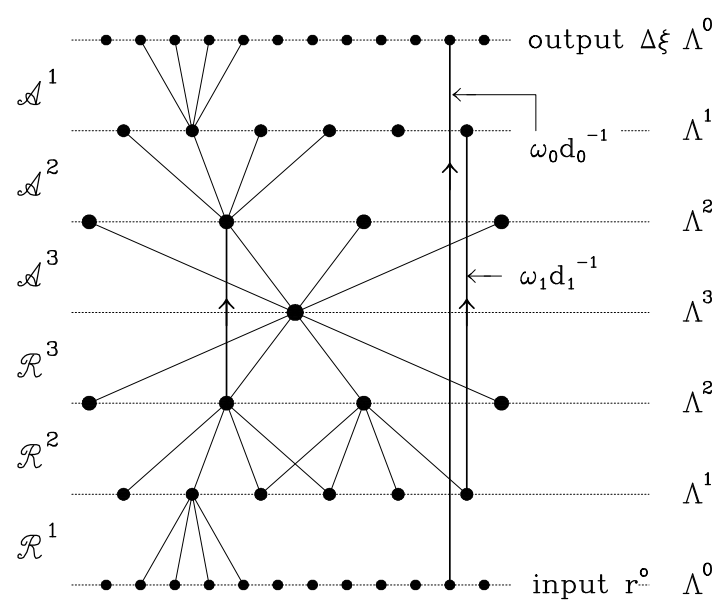

Figure 1. A feed-forward NMG architecture.

function of $r^{0}$. Except on the bottom layer, each node receives as input a weighted sum of the output of those nodes below it in the diagram to which it is connected. The weights are given by the connection strengths. Our neurons are linear because our problem is linear. The output of each neuron is a linear function of the input.

The result of the computation is

$\delta \xi=\left(\omega_{0} d_{0}^{-1}+\sum_{k \geq 1} \mathcal{A}^{[0 k]} \omega_{k} d_{k}^{-1} R^{[k 0]}\right) r^{0}$

where $R^{[k 0]}=R^{k} \ldots R^{2} R^{1}$ and $\mathcal{A}^{[0 k]}=\mathcal{A}^{1} \mathcal{A}^{2} \ldots \mathcal{A}^{k}$. The operators $C^{j}$ and $D_{j}(j>0)$, and the damping parameters $\omega_{j}(j>0)$ are not needed separately since they only enter in the combination $R^{j}=C^{j}\left(1-\omega_{j-1} D_{j-1} d_{j-1}^{-1}\right)$. The fundamental differential operator $D_{0}$ and its diagonal part $d_{0}$ are furnished as part of the problem. The connection strengths ("synaptical strengths") $\mathcal{A}_{z x}^{j}, R_{x z}^{j}$ (and possibly $\omega_{0}$ ) need to be found by a learning process in such a way that the actual output is as close as possible to the desired output. In supervised learning [7], pairs $\left(\xi^{\mu}, \zeta^{\mu}\right)$ ("training patterns") are presented to an ANN. Given input $\xi^{\mu}$, the actual output $O^{\mu}$ is compared to the target $\zeta^{\mu}$, and the connection strengths are adjusted in such a way that the cost functional $E=\sum_{\mu}\left\|O^{\mu}-\zeta^{\mu}\right\|^{2}$ gets minimized. An iter- 
ative procedure to achieve this minimization is called learning rule.

Taking for the sequence $\xi^{\mu}$ a complete orthonormal system of functions on $\Lambda^{0}$, in the limit $f^{0} \mapsto 0$, the target is $\zeta^{\mu}=0$ for any input, and the output $O^{\mu}=\varrho \xi^{\mu}$ by $($ (1). The learning rule for the resulting cost functional

$E=\sum_{\mu}\left\|\varrho \xi^{\mu}\right\|^{2}=\operatorname{tr} \varrho \varrho_{*} \equiv\|\varrho\|^{2} \stackrel{!}{=} \min$

is our previous optimality condition for multigrid relaxation in sect. 1 .

\section{LEARNING RULE PERFORMANCE}

The variant (ii) in sect. 2 involves a slightly different NMG. Instead of the connections between neighbouring layers of the multigrid, we now have connections from $\Lambda^{0}$ to $\Lambda^{k}$ with strength $C_{x z}^{[k 0]}$, and from $\Lambda^{j}$ to $\Lambda^{0}$ with strength $\mathcal{A}_{z x}^{[0 j]}$. If we adopt variational coarsening, all connection strengths are determined by interpolation kernels $\mathcal{A}^{[0 k]}$ which have to be learned. The damping factors $\omega_{k}$ were set to 1 and $d_{k}$ is the diagonal part of $D_{k}$ as before, with

$D_{k}=\mathcal{A}^{[0 k] *} D_{0} \mathcal{A}^{[0 k]}$.

The learning rule (ii) requires a process of "hard thinking" by the NMG. Nodes which have learned their lesson already - i.e. which have their connection strengths fixed - are used to instruct the rest of the neural net, adjusting the strengths of the next layer of nodes in the NMG.

A variant of this algorithm was tested in 2 dimensions, using $\mathrm{SU}(2)$-gauge fields which were equilibrated with standard Wilson action at various values of $\beta$, and $D_{0}=-\Delta-\varepsilon_{0}+\delta m^{2}$. $\varepsilon_{0}$ is the lowest eigenvalue of the covariant Laplacian $-\Delta$, and $\delta m^{2}>0$. Conventional relaxation algorithms for solving (1D) suffer from CSD for such $D_{0}$, for any volume and small $\delta m^{2}$.

It turned out that it was not necessary to find accurate solutions of the eigenvalue equation for the interpolation kernels $\mathcal{A}^{[0 j]}$. An approximation $\mathcal{A}_{z x}^{[0 j]}$ to $(-\Delta)^{-n} \delta_{z x}$ was computed by multigrid iteration. It does not depend on $\delta m^{2}$. Updating $\xi$ at $x \in \Lambda^{j}$ changes $\xi$ by

$\delta \xi_{z}=\mathcal{A}_{z x}^{[0 j]} d_{j, x}^{-1} r_{x}^{j}, \quad r^{j}=\mathcal{A}^{[0 j] *} r^{0}$.
The convergence rate (in units of MG iterations) of the $\xi$-iteration is shown in Fig. 2 for $\beta=1.0$. One MG iteration involved one sweep (in checkerboard fashion) through each MG layer, starting with $j=0$.

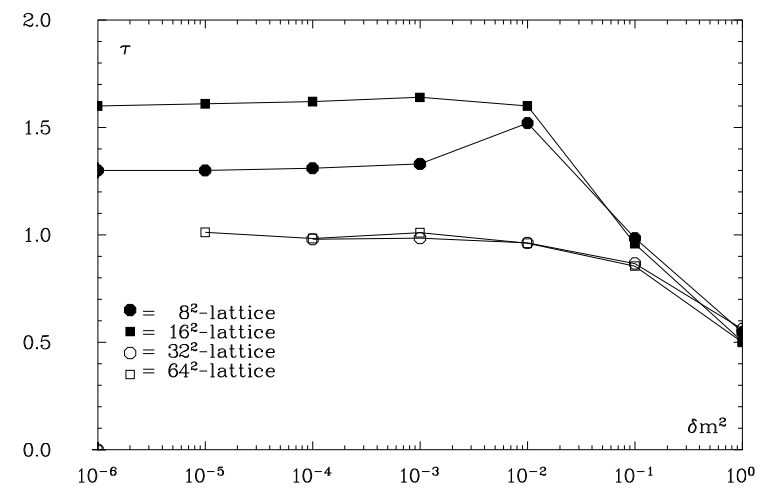

Figure 2. Correlation time $\tau$ as function of the lowest eigenvalue $\delta m^{2}$ in a representative gauge field configuration equilibrated at $\beta=1.0$. For the $64^{2}$ lattice, $\tau$ fluctuates very little with the gauge field.

We thank the HLRZ Jülich for computer time and assistance.

Support by Deutsche Forschungsgemeinschaft is gratefully acknowledged.

\section{REFERENCES}

1. G. Mack, in: Nonperturbative quantum field theory, eds. G. 't Hooft et al. (Plenum, New York 1989).

2. J. Goodman and A.D. Sokal, Phys. Rev. D 40 (1989) 2035.

3. W. Hackbusch, Multi-Grid Methods and Applications (Springer-Verlag, Berlin, 1985).

4. R.C. Brower, K. Moriarty, C. Rebbi and E. Vicari, Nucl. Phys. B 20 (1991) 89.

5. A. Hulsebos, J. Smit and J.C. Vink, Nucl. Phys. B 368 (1992) 379.

6. T. Kalkreuter, Phys. Lett. B 276 (1992) 485. 
7. J. Hertz, A. Krogh and R.G. Palmer, Introduction to the Theory of Neural Computation (Addison-Wesley, Redwood City CA, 1991). 